\title{
GAUGE THEORY OF DISLOCATION MELTING
}

\author{
H. KLEINERT \\ Institut für Theoretische Physik, Freie Universität Berlin, 1000 Berlin 33, Arnimallee 3, Germany
}

Received 4 December 1981

Revised manuscript received 14 January 1982

\begin{abstract}
We present a gauge theory for an ensemble of dislocation lines with elastic interactions as a model of melting. There is close similarity with the Ginzburg-Landau theory of superconductivity, except that in our theory order parameter and magnetic potential describe translational disorder and stress, respectively, and that the temperature axis is reversed. Above a critical temperature, the disorder proliferates, thereby rendering a finite penetration depth to stress as a signal for the molten state.
\end{abstract}

Since Shockley's original suggestion [1] it is widely believed [2-5] that one of the main causes for the breakdown of crystalline order during the process of melting is the sudden proliferation of line-like defects of the translational type, called dislocations. Such a process would liberate the translational movement of the lat tice constituents along the basis vectors of the crystal. Once this happens, the formation of orientational defects called disclinations, can take place at a much lower cost in energy than in the crystal. Their proliferation establishes complete isotropy and the final state is indeed a proper liquid [4].

It is the purpose of this note to present a field theory by which the study of translational melting becomes technically quite simple due to its similarity with the Ginzburg-Landau theory of superconductivity. We shall proceed by considering dislocation lines as random chains and develop a complex scalar field theory describing grand-canonical ensembles of these. After this, elasticity is formulated in terms of a gauge potential which may be coupled to the scalar fields in a locally gauge invariant way. This procedure is shown to give the correct elastic energy. The resulting gauge theory has simple properties permitting a straightforward discussion of the melting process in analogy with the phase transition in superconductors.

A random chain whose links have a length $l$ and are labelled by the length parameter $s$ has a gaussian link-to-link probability distribution [6]

$P\left(\boldsymbol{x}^{\prime}-\boldsymbol{x}, s^{\prime}-s\right)=\left[2 \pi\left(s^{\prime}-s\right) l / 3\right]^{-3 / 2} \exp \left[-3\left(\boldsymbol{x}^{\prime}-\boldsymbol{x}\right)^{2} / 2 l\left(s^{\prime}-s\right)\right]$,

which agrees with the correlation function of a free non-relativistic particle of mass $M=3 / l$ as a function of time $t$ if this time is continued to imaginary values $t=-\mathrm{i}$ s. For a grand-canonical ensemble of such random chains we may therefore use a second quantized field $\psi(x, t)$ with a lagrangian

$\mathcal{L}(\boldsymbol{x}, t)=\psi^{+}(\boldsymbol{x}, t)\left[\mathrm{i} \partial_{t}+(1 / 2 M) \nabla^{2}\right] \psi(\boldsymbol{x}, t)$,

whose propagator

$\left.\left\langle\hat{T}_{t} \psi\left(\boldsymbol{x}^{\prime}, t^{\prime}\right) \psi^{+}(\boldsymbol{x}, t)\right\rangle\right|_{t^{\prime}-t=-\mathrm{i}\left(s^{\prime}-s\right)}=\int \frac{\mathrm{d}^{3} k}{(2 \pi)^{3}} \exp \left[\mathrm{i} \boldsymbol{k}\left(\boldsymbol{x}^{\prime}-\boldsymbol{x}\right)-\left(k^{2} / 2 M\right)\left(s^{\prime}-s\right)\right]$

coincides directly with (1). The field has a divergenceless current

$j(\boldsymbol{x})=\int \mathrm{d} t \psi^{+}(\boldsymbol{x}, t) \frac{1}{2 M \mathrm{i}} \overleftrightarrow{\nabla} \psi(\boldsymbol{x}, t)$, 
which is the randomly fluctuating second quantized version of a single localized line singularity along a path $\boldsymbol{x}(s)$ : $j(x)=\oint \mathrm{d} s x^{\prime}(s) \delta^{(3)}(x-x(s))$. For the process of melting, the information on the length parameter $s$ will be superfluous.

Instead of (1) we may study the probability that a random chain with arbitrary length passes through $\boldsymbol{x}$ and $\boldsymbol{x}^{\prime}$. The distribution of length is ruled by a chemical potential $\lambda$ and the probability becomes

$P\left(x^{\prime}-x\right)=\int_{0}^{\infty} \mathrm{d}\left(s^{\prime}-s\right) P\left(x^{\prime}-x, s^{\prime}-s\right) \exp \left[-(\lambda / T)\left(s^{\prime}-s\right)\right]$.

The chemical potential has two terms and may be written as $\lambda=\lambda_{\text {core }}-w T$, with $\lambda_{\text {core }}$ accounting for the core energy and $w$ being the entropy per link ( $\mathrm{e}^{-w l}=6$ counts the number of link connections to the next neighbor). Inserting for $P\left(x^{\prime}-x, s^{\prime}-s\right)$ the form (3) this gives

$P\left(x^{\prime}-x\right)=2 M \int \frac{\mathrm{d}^{3} k}{(2 \pi)^{3}}\left(k^{2}+m^{2}\right)^{-1} \mathrm{e}^{\mathrm{i} k\left(x^{\prime}-x\right)}, \quad m^{2}=(\lambda / T) 2 M$,

which is nothing but the correlation function of a Klein-Gordon field of mass $m$ whose energy density reads

$f_{0}(\boldsymbol{x})=T\left[\nabla \varphi^{+}(\boldsymbol{x}) \nabla \varphi(\boldsymbol{x})+m^{2} \varphi^{+}(\boldsymbol{x}) \varphi(\boldsymbol{x})\right]$.

The divergenceless current $j=(T / 2 \mathrm{i}) \varphi+\stackrel{\leftrightarrow}{\nabla} \varphi$ is the analogue of (4) with the length parameter $s$ averaged out. The temperature factor $T$ exhibits the purely stochastic and entropic nature of the $\varphi(x)$ field. Indeed, were it not for the core energy $\lambda_{\text {core }}$ the $\varphi$ fluctuations in the partition function

$Z=\int D \varphi D \varphi^{+} \exp \left(T^{-1} \int \mathrm{d}^{3} x f_{0}(x)\right)$

would be temperature independent.

The fields $\varphi(x)$ are now identified with the dislocation lines of any length passing through $\boldsymbol{x}$. Apart from their spatial distribution, they are characterized by a Burgers vector $b$ which has the same additivity properties as a charge: two dislocation loops may annihilate each other if they have opposite Burgers vectors and a line may branch off into several others as long as the sum of Burgers vectors remains invariant. Lines with higher Burgers vectors may be generated as bound states of lower ones in just the same way as nuclei are built from protons and neutrons. Thus we may confine our attention to an energy density $f_{0}(\boldsymbol{x})$ of the form (5) which contains the fields of only the fundamental Burgers vectors $b^{(\alpha)}$, as long as we add an interaction capable of generating composite dislocation lines. As far as melting is concerned, we shall use the simple approximation

$f_{\mathrm{int}} \approx \sum_{\alpha, \alpha^{\prime}} g_{\alpha \alpha^{\prime}}\left|\varphi_{\alpha}(x)\right|\left|\varphi_{\alpha^{\prime}}(x)\right|^{2}$

The conservation of Burgers vectors is manifest by $f=f_{0}+f_{\text {int }}$ being invariant under overall phase changes

$\varphi_{\alpha}(\boldsymbol{x}) \rightarrow \exp \left(-\mathrm{i} \boldsymbol{b}^{(\alpha)} \mathbf{\Lambda}\right) \varphi_{\alpha}(\boldsymbol{x})$.

It appears to be one of the fundamental principles of physical theories that whenever there is a conserved quantum number whose conservation is enforced by phase transformations (7), there is also a gauge field which makes the energy density invariant under the local generalization of (7): $\varphi_{\alpha}(\boldsymbol{x}) \rightarrow \exp \left[-\mathrm{i} \boldsymbol{b}^{(\alpha)} \boldsymbol{\Lambda}(\boldsymbol{x})\right] \varphi_{\alpha}(\boldsymbol{x})$. This principle has guided the construction of all modern theories of weak, electromagnetic, and strong interactions and it is gratifying to discover its validity also in the present case. Here it is the long-range interactions among dislocation lines due to linear elasticity which lead to local gauge invariance.

Consider the elastic energy $f_{\mathrm{el}}(\boldsymbol{x})=(4 \mu)^{-1}\left\{\sigma_{i j}^{2}-[\nu /(1+\nu)] \sigma_{i i}^{2}\right\}$ where $\mu, \nu$ are the well-known elastic constants [7]. The stress tensor $\sigma_{i j}$ is symmetric and divergenceless. Therefore it can be expressed in terms of another symmetric tensor $h_{i j}(x)$ as $\sigma_{i j}(x)=\epsilon_{j k l} \nabla_{k} \epsilon_{i m n} \nabla_{m} h_{l n}(x)$. This representation is invariant under the local gauge trans- 
formations $h_{l n}(x) \rightarrow h_{l n}(x)+\nabla_{l} \xi_{n}(x)+\nabla_{n} \xi_{l}(x)$. For the sake of maximal analogy with magnetism we find it convenient to introduce the field $A_{l}^{i}(\boldsymbol{x})=\epsilon_{i m n} \nabla_{m .} h_{l n}(\boldsymbol{x})$ as a stress potential with the property $\nabla_{i} A_{l}^{i}(\boldsymbol{x})=0, A_{i}^{i}=0$. The gauge transformations become $A_{l}^{i}(\boldsymbol{x}) \rightarrow A_{l}^{i}(\boldsymbol{x})+\nabla_{l} \Lambda^{i}(\boldsymbol{x})$ with $\Lambda^{i}(\boldsymbol{x})=\epsilon_{i m n} \nabla_{m} \xi_{n}(\boldsymbol{x})$ being arbitrary functions satisfying the transversality condition $\nabla_{i} \Lambda^{i}(\boldsymbol{x})=0$. In terms of $A_{l}^{i}(\boldsymbol{x})$, the elastic energy reads

$f_{\mathrm{e} 1}(x)=\frac{1}{4 \mu} \sum_{k} k^{2} A_{l}^{i}(-k)\left(P^{(2,-2)}+P^{(2,2)}+\frac{1-\nu}{1+\nu} P^{(1,0)}\right)_{i l, i^{\prime} l^{\prime}} A_{l^{\prime}}^{i^{\prime}}(k)$

where $P^{(s, h)}(\hat{k})$ are the projection matrices into spin $s$ helicity $h$ combinations of the matrix $A_{l}^{i}(k)$. The advantage of the stress potential $A_{l}^{i}$ is that the elastic energy of a dislocation loop can be calculated from a local coupling in complete analogy with the magnetic energy of a current loop: If $u_{i}^{\mathrm{I}}$ is the displacement vector due to a dislocation loop, its energy in a stress field $\sigma_{i j}^{\mathrm{II}}$ is given by

$E=\int \mathrm{d}^{3} x \sigma_{i j}^{\mathrm{II}} \nabla_{j} u_{i}^{\mathrm{I}}=\int \mathrm{d}^{3} x \nabla_{j}\left(\sigma_{i j}^{\mathrm{II}} u_{i}^{\mathrm{I}}\right)=b_{i} \int \mathrm{d} S_{j} \sigma_{i j}^{\mathrm{II}}$,

where we have used the fact that $u_{i}^{I}$ has a discontinuity $\Delta u_{i}^{\mathrm{I}}=b_{i}$ over some surface spanning the dislocation loop. But writing $\sigma_{i j}$ as the curl of $A_{l}^{i}$, this becomes a contour integral over the loop:

$E=b_{i} \int \mathrm{d} S_{j} \epsilon_{j k l} \nabla_{k} A_{l}^{i}=b_{i} \int \mathrm{d} s x_{l}^{\prime}(s) A_{l}^{i}(x(s))=b_{i} \int \mathrm{d}^{3} x j_{l}(\boldsymbol{x}) A_{l}^{i}(\boldsymbol{x})$.

This local coupling is obviously gauge invariant (due to $\nabla_{l} j_{l}(\boldsymbol{x})=0$ ) and differs from magnetic systems, where $E=I \int \mathrm{d}^{3} x j_{l}(\boldsymbol{x}) A_{l}(\boldsymbol{x})$ by having three vector potentials $A_{l}^{1}, A_{l}^{2}, A_{l}^{3}$, each having its own "current" $b_{1}, b_{2}, b_{3}$.

It may be easily verified that the coupling $(9)$ reproduces the correct elastic energy between two dislocation lines with the correlation function, due to (8),

$\left\langle A_{l}^{i}\left(\boldsymbol{x}^{\prime}\right) A_{l^{\prime}}^{i^{\prime}}(\boldsymbol{x})\right\rangle=2 \mu T \int \frac{\mathrm{d}^{3} k}{(2 \pi)^{3}} \mathrm{e}^{\mathrm{i} k\left(\boldsymbol{x}^{\prime}-x\right)} k^{-2}\left(P^{(2,-2)}+P^{(2,2)}+\frac{1+\nu}{1-\nu} P^{(1,0)}\right)_{i l, i^{\prime} l^{\prime}}$,

in agreement with Blin's conventionally derived formula [8]. We can now introduce the same local coupling into the field theory by the standard minimal replacement and find

$f(\boldsymbol{x})=T \sum_{\alpha}\left\{\left|\left[\nabla_{l}-(\mathrm{i} / T) b_{i} A_{l}^{i}\right] \varphi_{\alpha}\right|^{2}+m_{\alpha}^{2}\left|\varphi_{\alpha}\right|^{2}\right\}+\sum_{\alpha, \alpha^{\prime}} g_{\alpha \alpha^{\prime}}\left|\varphi_{\alpha}\right|^{2}\left|\varphi_{\alpha^{\prime}}\right|^{2}+f_{\mathrm{cl}}$

as the correct theory for a grand-canonical ensemble of interacting dislocation lines of arbitrary lengths. The theory displays manifest local gauge invariance under transformations

$\varphi_{\alpha}(\boldsymbol{x}) \rightarrow \exp \left[-\mathrm{i} \boldsymbol{b}^{(\alpha)} \boldsymbol{\Lambda}(\boldsymbol{x})\right] \varphi_{\alpha}(\boldsymbol{x}), \quad A_{l}^{i}(\boldsymbol{x}) \rightarrow A_{l}^{i}(\boldsymbol{x})+\nabla_{l} \Lambda^{i}(\boldsymbol{x}), \quad \boldsymbol{\nabla} \boldsymbol{\Lambda}(\boldsymbol{x})=0$.

Due to the similarity with the Ginzburg-Landau theory it is rather straightforward to extract some basic physical properties: For high enough temperature, $T>T_{\alpha, \mathrm{c}}=\lambda_{\alpha, \mathrm{core}} / w_{\alpha}$, the chemical potentials $\lambda_{\alpha}$ change sign and the fluctuations of the $\varphi_{\alpha}$ fields around $\varphi_{\alpha}=0$ diverge. The quartic potential, however, prevents a catastrophy and stabilizes at certain non-zero values $\varphi_{\alpha}=\left|\varphi_{\alpha}\right| \mathrm{e}^{-\mathrm{i} \gamma_{\alpha}}$. This signalizes the proliferation of dislocation lines. At the same time, the fluctuations of the gauge field receive an additional quadratic term $T^{-1} \Sigma_{\alpha} b_{i}^{(\alpha)} b_{l}^{(\alpha)}\left|\varphi_{\alpha}\right|^{2} A_{l}^{i} A_{l}^{j}$ which in the case of a simple cubic crystal can be averaged to $(4 \mu)^{-1} m_{\mathrm{A}}^{2} A_{l}^{i} A_{l}^{i}$ with $m_{\mathrm{A}}^{2}=4 \mu\left|\boldsymbol{b}^{(\alpha)}\right| 2\left|\varphi_{\alpha}\right|^{2} / T$. This is a mass term which limits the penetration of the stress potential to a finite depth $m_{\mathrm{A}}^{-1}$, a characteristic of the liquid phase. In the present description, this is completely analogous to the way in which order prevents the invasion of a magnetic field into a superconductor (only that there, order proliferates at low, here, disorder at high temperature).

The question arises as to the propagation of sound waves in this description of the liquid phase. This can be answered by noting that in the absence of a gauge potential, the disordered phase would be characterized by long- 
range oscillations of the phase $\gamma_{\alpha}(x)$ of $\varphi_{\alpha}(x)$ (Nambu-Goldstone bosons). As the gauge field is turned on, part of these phase degrees of freedom become unphysical since they can be removed by a gauge transformation. In fact, we see above that phase oscillation $\gamma_{\alpha}(x)$ with $b^{(\alpha)}$ pointing transversal to the momentum direction can be absorbed. The longitudinal parts of $\gamma_{\alpha}(x)$, however, survive as a physical degree of freedom, and may be identified with the sound excitation in the molten phase ("hot sound" as the disorder analogue of "zero sound").

Of sourse this simple mean-field discussion requires fluctuation corrections. Since the gauge field appears quadratically in $f$ its fluctuations can directly be integrated in the partition function. For constant $\left|\varphi_{\alpha}\right|, f$ receives an additional "black body" energy of the form

$$
\begin{aligned}
f_{\mathrm{bb}} & =\frac{T}{2} \int \frac{\mathrm{d}^{3} k}{(2 \pi)^{3}}\left[2 \log \left(k^{2}+m_{\mathrm{A}}^{2}\right)+\log \left(k^{2}+\frac{1+\nu}{1-\nu} m_{\mathrm{A}}^{2}\right)\right] \\
& =\frac{1}{2 \pi^{2}}\left\{\frac{1}{2} k_{\mathrm{c}}^{3}\left(\log k_{\mathrm{c}}-\frac{2}{3}\right)+k_{\mathrm{c}}\left(1+\frac{1}{2} \frac{1+\nu}{1-\nu}\right) m_{\mathrm{A}}^{2}-\frac{\pi}{3}\left[1+\frac{1}{2}\left(\frac{1+\nu}{1-\nu}\right)^{3 / 2}\right] m_{\mathrm{A}}^{3}\right\} .
\end{aligned}
$$

The first term is trivial and the second merely renormalizes $m_{\alpha}^{2}$, which amounts to a shift in $T_{\alpha, \mathrm{c}}$.

The third cubic term, however, has the consequence that the transition occurs at a precocious value of $m_{\alpha}^{2}=$ $m_{\alpha, \text { prec }}^{2}=2\left(3 \pi^{2} T^{2}\langle g\rangle\right)^{-1}\left(\mu b^{(\alpha) 2}\right)^{3}$ before the $m_{\alpha}^{2}\left|\varphi_{\alpha}\right|^{2}$ term loses its stability. At that value, $\varphi_{\alpha}$ jumps discontinuously from zero to $\varphi_{\alpha} \neq 0$ and this jump is accompanied by a latent heat. Thus the transition is of first order. This effect of fluctuations has been recognized in the Ginzburg-Landau theory for a long time [9] but was never confirmed experimentally. This is due to the fact that the assumption of constant $\varphi_{\alpha}$ is acceptable only in a type I superconductor where $m_{\alpha, \text { prec }}^{2}$ is large. In the type II case, $\varphi_{\alpha}$ fluctuates too much to permit an integration over the $A_{l}^{i}$ fluctuations. For dislocations, the parameter distinguishing type I and type II is the ratio of elastic and steric repulsion energy, $\kappa=\left(\langle g\rangle / \mu b^{2}\right)^{1 / 2}$. For $\kappa \gtrless 1 / \sqrt{ } 2$ the solid is of type $\left\{{ }_{I}^{\text {II }}\right\}$. The range $\left|m_{\alpha}^{2}\right|>m_{\alpha, \mathrm{L}}^{2}$, where fluctuations are small is given by the Landau criterium, here $m_{\alpha, \mathrm{L}}^{2} \approx \kappa^{6} m_{\alpha, \mathrm{prec}}^{2}$. Thus, for small $k, m_{\alpha, \mathrm{prec}}^{2} \gg m_{\alpha, \mathrm{L}}^{2}$ and the evaluation (12) is reliable making the transition first order.

The largeness of $m_{\alpha, \text { prec }}^{2}$ is observed experimentally in the form of a high transition heat $\Delta Q \approx(\mu T /\langle g\rangle) m_{\alpha, \text { prec }}^{4}$ Within the present theory, this is taken as evidence for the smallness of the steric repulsion between the dislocation lines. Notice that were it not for the largeness of $m_{\alpha, \text { prec }}^{2}$, melting would occur at a much higher temperature, close to the purely entropic values $T_{\alpha, \mathrm{c}}=\lambda_{\alpha, \text { core }} / w_{\alpha}$.

Of course, the same type of field theory can also be used for the vortex lines in superfluid ${ }^{4} \mathrm{He}$ only that there the Burgers "vectors" are scalars and there is just one gauge potential $A_{l}$ with an elastic energy $f_{\mathrm{el}}=\frac{1}{2} \rho_{\mathrm{s}}(\nabla \times A)^{2}$, just as in magnetism. The question why the transition in ${ }^{4} \mathrm{He}$ remains of second order, in spite of the gauge field fluctuations, finds its answer in the fact that here the steric repulsion is large enough to make the theory of type II.

It may be worth contrasting the present theory with existing applications of the Landau expansion to melting [10]. There, the system is described in terms of an orientational tensor order parameter, say $\varphi_{i j k l}$, which takes a non-zero value in the solid phase (i.e. for $T<T_{\mathrm{c}}$ ). If the phase transition were of second order, Landau's phenomenological expansion could be truncated after the quartic term and would give a rather complete explanation of physical observables in the immediate neighborhood of $T_{\mathrm{c}}$. However, experimentally it is of first order and the expansion always contains a cubic term, on symmetry grounds. This, unfortunately, destroys the applicability of Landau's approach: first, the expansion can no longer be truncated and second, the transition loses its universality character and is no longer independent of the physical processes even right at the transition temperature.

The defect model advanced by ref. [4] (inspired by ref. [3]) does, in fact, introduce physical processes, which might lead to a satisfactory description of the first order transition. Their treatment in 3 dimensions (in the second of refs. [4]) has, however, two drawbacks:

(i) It is based on Blin's formula (the analogue of Biot-Savart's law) between dislocation lines which is of the "action-at-a-distance" form and therefore clumsy to handle. The simplicity and beauty of Maxwell's theory is rooted in it being a local field theory which has become the prototype for all fundamental theories of matter. Our 
theory is the analogue of Maxwell's.

(ii) Blin's formula has a meaning only for isolated dislocation lines in a crystal at zero temperature. Our energy (11) gives a partition function

$Z=\int D \varphi D \varphi^{+} D A \exp \left(-\frac{1}{T} \int \mathrm{d}^{3} x f(x)\right)$

which deals with a grand canonical ensemble of dislocation lines in thermal equilibrium with lattice vibrations at any temperature. This partition function has a chance of applying also to the liquid phase which is densely filled with strongly fluctuating dislocations in which there is positional chaos and no way of employing Blin's formula. In our theory this situation is simply accounted for by $\langle\varphi\rangle \neq 0$.

After this manuscript was submitted, further developments have taken place. Disclinations were included into the gauge field theory [11], connections between quark confinement and melting were pointed out [12], an explicit construction of fluctuating defect lines was given on a lattice [13] and a geometric description of stresses and defects was proposed [14]. A summaty of all these results is in preparation [15] ${ }^{\dagger 1}$.

In the course of this work it has become apparent that our way of dealing with the non-linearities of crystal forces may have implications upon the physical interpretation of the symmetry breaking Higg's particles in the unified gauge theory of weak and electromagnetic interactions which many high-energy laboratories are now searching for. Rather than being new fundamental objects they may be defects in the non-linear $W$-meson fields which mediate the interactions [16].

The author thanks W. Helfrich and W. Müller for stimulating discussions.

$\$ 1$ A preliminary account of our theory was presented at the International Conference of the European Physical Socicty in Lisbon, July 1981 .

\section{References}

[1] W. Shockley, L'Etat solide (Institut International de Physique Solvay, Brussels, 1952) p. 431.

[2] For earlier work see the review by R.M.J. Cotterill, in: Ordering in strongly fluctuating condensed matter systems, ed. Tormond Riste (Plenum, New York, 1980).

[3] J.M. Kosterlitz and D.J. Thouless, J. Phys. C6 (1973) 1181; Prog. Low Temp. Phys. 7B (1978) 371.

[4] B.I. Halperin and D.R. Nelson, Phys. Rev. Lett. 41 (1978) 121 ;

D.R. Nelson and J. Toner, Phys. Rev. B24 (1981) 363 and references therein.

[5] S.F. Edwards and M. Warner, Philos. Mag. 40 (1979) 257.

[6] H. Yamakawa, Modern theory of polymer solutions (Harper and Row, New York, 1971).

[7] L.D. Landau and E.M. Lifshitz, Theory of elasticity, (Pergamon, New York, 1959).

[8] J. Blin, Acta Mech. 3 (1955) 199.

[9] B.H. Halperin, T.C. Lubensky and S.K. Ma, Phys. Rev. Lett. 32 (1974) 292.

[10] S. Alexander and J.P. McTague, Phys. Rev. Lett. 40 (1978) 702;

S. Hess, Z. Naturforsch. 35A (1979) 69.

[11] H. Kleinert, Lett. Nuovo Cimento, to be published.

[12] H. Kleinert, Phys. Lett. B.

[13] H. Kleinert, Phys. Lett. A.

[14] H. Kleinert, Lett. Nuovo Cimento.

[15] H. Kleinert, Fortschr. Phys.

[16] H. Kleinert, Phys. Lett. B. 